\title{
Theoretical studies of the electron paramagnetic resonance parameters and local structures for $\mathrm{Cu}^{2+}$ in $(\mathbf{1 0 0 - 2}) \mathrm{TeO}_{2-x} \mathrm{Ag}_{2} \mathrm{O}_{-x} \mathrm{WO}_{3}$ glasses
}

\author{
H.-N. Dong and R. Zhang* \\ College of Physics and Engineering, \\ Chengdu Normal University, Chengdu 611130, P.R. China. \\ *e-mail: zhangrongcdnu@163.com
}

Received 26 July 2020; accepted 17 September 2020

\begin{abstract}
The electron paramagnetic resonance (EPR) parameters and local structures for $\mathrm{Cu}^{2+}$ in $(100-2 x) \mathrm{TeO}_{2-x} \mathrm{Ag}_{2} \mathrm{O}_{-x} \mathrm{WO}_{3}(\mathrm{TAW})(7.5 \leq x \leq$ $30 \mathrm{~mol} \%$ ) glasses are quantitatively studied for distinct modifier concentrations $x$. The octahedral $\mathrm{Cu}^{2+}$ centers are subject to the medium tetragonal elongations of about $2 \%$ along the $\mathrm{C}_{4}$ axis due to the Jahn-Teller effect. By utilizing only three adjusted coefficients $a, b$, and $\omega$, the quantities $\left(D_{q}, k, \tau\right.$, and $\left.\kappa\right)$ can be suitably characterized by the Fourier type functions, which reasonably account for the experimental concentration dependences of the d-d transition bands and EPR parameters. The calculation results are discussed, and the mechanisms of the above concentration dependences of these quantities are illustrated by the modifications of the local structures and the electron cloud distribution around the $\mathrm{Cu}^{2+}$ dopant with the variations of the concentration $x$.
\end{abstract}

Keywords: $\mathrm{Cu}^{2+} ; \mathrm{TeO}_{2-x} \mathrm{Ag}_{2} \mathrm{O}_{-x} \mathrm{WO}_{3}$ glasses; defect structures; electron paramagnetic resonance.

PACS: 76.30.Fc; 75.10.Dg

DOI: https://doi.org/10.31349/RevMexFis.67.1

\section{Introduction}

Tellurite silver $\left(\mathrm{TeO}_{2}-\mathrm{Ag}_{2} \mathrm{O}\right)$ glasses exhibit useful dielectric [1], electrical [2], structural [3], thermal and Raman [4] and non-linear optical properties [5,6]. Similarly, tellurite tungsten $\left(\mathrm{TeO}_{2}-\mathrm{WO}_{3}\right)$ glasses can provide unique electrical [7], linear optical [8], and luminescent properties for green laser [9] as well as efficient radiation shielding applications [10-12]. Thus, it is expected that a combination of the above glasses as tellurite silver tungsten $\left(\mathrm{TeO}_{2}-\mathrm{Ag}_{2} \mathrm{O}-\mathrm{WO}_{3}\right.$ or TAW) systems may enhance the above properties and applications with two modifiers $\mathrm{Ag}_{2} \mathrm{O}$ and $\mathrm{WO}_{3}$. In general, the above aspects of TAW glasses can be strongly relied on the local structures of the network of the glass systems, and they can be efficiently analyzed by means of the electron paramagnetic resonance (EPR) technique. As a popular dopant in oxide glasses, $\mathrm{Cu}^{2+}$ is widely used to probe the local structural properties due to the prominent EPR signals recordable at room temperature. Recently, d-d transition optical absorption and EPR experiments were performed for (100$2 \mathrm{x}) \mathrm{TeO}_{2-x} \mathrm{Ag}_{2} \mathrm{O}_{-x} \mathrm{WO}_{3}(7.5 \leq x \leq 30$ mol \%) glasses with $1 \mathrm{~mol} \% \mathrm{CuO}$ dopants [13]. The measured EPR parameters ( $g$ factors $g_{\|}$and $g_{\perp}$ and hyperfine structure constants $A_{\|}$) were analyzed for the various $\mathrm{WO}_{3}$ or $\mathrm{Ag}_{2} \mathrm{O}$ concentrations by using the simple $g$ formulas and some adjustable bonding parameters $\alpha^{2}, \beta_{1}^{2}$, and $\beta^{2}$ (which are largely higher than unity) [13]. However, the defect structure around impurity $\mathrm{Cu}^{2+}$ in TAW glasses and its concentration dependence has not been theoretically obtained up to now. So, the concentration variations of the EPR parameters and local structures in the vicinity of the copper dopants in TAW glasses, as well as their mechanisms, need to be further studied uniformly.

This article aims to perform theoretical calculations of the EPR parameters and local structures for $\mathrm{Cu}^{2+}$ in TAW glasses at distinct $\mathrm{WO}_{3}$ or $\mathrm{Ag}_{2} \mathrm{O}$ concentrations $\mathrm{x}$ based on the perturbation formulas of $g$ factors and hyperfine structure constants for a $3 \mathrm{~d}^{9}$ ion in tetragonally elongated octahedra. And the features of EPR parameters and local environments around the copper dopants are also analyzed because of the modifications of the local crystal-field strength and electron cloud distributions with the increase of the modifier concentration.

\section{Theory and calculation}

In the light of the observed $g$ factors $\left(2<g_{\perp}<g_{\|}\right.$[13] $)$ for $\mathrm{Cu}^{2+}$ in TAW glasses at all concentrations $\mathrm{x}$, the impurities $\mathrm{Cu}^{2+}$ are expected to locate at suitable octahedral sites, with the octahedral $\left[\mathrm{CuO}_{6}\right]^{10-}$ clusters showing the tetragonal elongation distortions due to the Jahn-Teller effect [1418]. For a tetragonally elongated $3 \mathrm{~d}^{9}$ cluster, the original lower two-fold orbitally degenerated irreducible representation $2 E_{g}$ of cubic symmetry may be separated into two orbital singlets ${ }^{2} B_{1 g}$ and ${ }^{2} A_{1 g}$, the former being the new ground state. The original upper three-fold orbitally degenerated representation ${ }^{2} T_{2 g}$ can split into an orbital doublet ${ }^{2} E_{g}$ and an orbital singlet ${ }^{2} B_{2 g}$ [16-18].

To study the EPR parameters and local structures for TAW: $\mathrm{Cu}^{2+}$ glasses, the perturbation formulas of these parameters for tetragonally elongated octahedral $3 d^{9}$ clusters can be adopted here. Thus, we have $[19,20]$ : 


$$
\begin{aligned}
g_{\|} & =g_{s}+\frac{8 k \zeta}{E_{1}}+\frac{k \zeta^{2}}{E_{2}^{2}}+\frac{4 k \zeta^{2}}{E_{1} E_{2}}-g_{s} \zeta^{2}\left[\frac{1}{\left(E_{1}^{2}\right)}-\frac{1}{\left(2 E_{2}^{2}\right)}\right] \\
& +\frac{k \zeta^{3}\left[\frac{4}{E_{1}}-\frac{1}{E_{2}}\right]}{E_{2}^{2}}-2 k \zeta^{3} \frac{\left(\frac{2}{E_{1} E_{2}}-\frac{1}{E_{2}^{2}}\right)}{E_{1}} \\
& +g_{s} \zeta^{3}\left[\frac{1}{\left(E_{1} E_{2}^{2}\right)}-\frac{1}{\left(2 E_{2}^{3}\right)}\right], \\
g_{\perp} & =g_{s}+\frac{2 k \zeta}{E_{2}}-\frac{4 k \zeta^{2}}{\left(E_{1} E_{2}\right)}+k \zeta^{2}\left[\frac{2}{\left(E_{1} E_{2}\right)}-\frac{1}{E_{2}^{2}}\right] \\
& +\frac{2 g_{s} \zeta^{2}}{E_{1}^{2}}+k \zeta^{3}\left(\frac{2}{E_{1}}-\frac{1}{E_{2}}\right)\left[\frac{\left(\frac{1}{E_{2}}+\frac{2}{E_{1}}\right)}{\left(2 E_{2}\right)}\right] \\
& +k \zeta^{3}\left[\frac{\left(\frac{1}{E_{1}^{2}}-\frac{1}{\left(E_{1} E_{2}\right)}+\frac{1}{E_{2}^{2}}\right)}{\left(2 E_{2}\right)}\right], \\
A_{\|} & =P\left[-\kappa-\frac{4 H}{7}+\left(g_{\|}-g_{s}\right)+\frac{3\left(g_{\perp}-g_{s}\right)}{7}\right] \\
A_{\perp} & =P\left[-\kappa-\frac{2 H}{7}+\frac{11\left(g_{\perp}-g_{s}\right)}{14}\right] .
\end{aligned}
$$

Here, $g_{s}(\approx 2.0023)$ is the pure spin $g$; value $k, \zeta$, and $P$ are, respectively, the orbital reduction factor, the spin-orbit coupling coefficient, and the dipolar hyperfine coupling parameter of the $3 \mathrm{~d}^{9}$ ion in glasses, in terms of the relationships, $\zeta=k \zeta_{0}$ and $P=k P_{0}$ with the corresponding free-ion values $\zeta_{0}$ and $P_{0}$ represents the core polarization constant, characteristic of the Fermi contact interactions related to the isotropic central ion $3 \mathrm{~d}-3 \mathrm{~s}(4 \mathrm{~s})$ orbital admixtures. $H$ denotes the reduction factor related to the anisotropic central ion $3 \mathrm{~d}-$ $3 \mathrm{~s}(4 \mathrm{~s})$ orbital admixtures due to the tetragonal elongation distortion [21].

In the above expressions, the energy separations $E_{1}$ and $E_{2}$ stand for the separations between the excited ${ }^{2} B_{2 g}$ and ${ }^{2} E_{g}$ states and the ground ${ }^{2} B_{1 g}$ state, which can be written in terms of the cubic crystal-field parameter $D q$ and the tetragonal crystal-field parameters $D_{s}$ and $D_{t}[17,18]$ :

$$
\begin{aligned}
& E_{1}=10 D q, \\
& E_{2}=10 D q-3 D s+5 D t .
\end{aligned}
$$

From the local structural properties of the studied $\left[\mathrm{CuO}_{6}\right]^{10-}$ clusters, the tetragonal crystal-field parameters can be determined from the superposition model [21-22]:

$$
\begin{aligned}
& D s=-\frac{4}{7} \bar{A}_{2}\left(R_{0}\right)\left[\left(\frac{R_{0}}{R_{\perp}}\right)^{t_{2}}-\left(\frac{R_{0}}{R_{\|}}\right)^{t_{2}}\right], \\
& D t=-\frac{16}{21} \bar{A}_{4}\left(R_{0}\right)\left[\left(\frac{R_{0}}{R_{\perp}}\right)^{t_{4}}-\left(\frac{R_{0}}{R_{\|}}\right)^{t_{4}}\right] .
\end{aligned}
$$

Here the superscripts $t_{2}(\approx 3)$ and $t_{4}(\approx 5)$ are the power-law exponents [21]. With the reference distance $R_{0}$, and are the intrinsic parameters, satisfying the relationships $\bar{A}_{4}\left(R_{0}\right) \approx(3 / 4) D q$ and the ratio $\bar{A}_{2}\left(R_{0}\right) / \bar{A}_{4}\left(R_{0}\right) \approx 9-12$ for octahedral $3 \mathrm{~d}^{n}$ groups [23-25]. And we take $\bar{A}_{2}\left(R_{0}\right) \approx$ $9 \bar{A}_{4}\left(R_{0}\right)$ here. For convenience, the defect structure near the impurity $\mathrm{Cu}^{2+}$ can be characterized as two longer axial copper-oxygen bonds and four shorter planar ones, corresponding to a relative tetragonal elongation ratio $\tau$ and the positive $\mathrm{g}$ anisotropy $\Delta g\left(=g_{\|}-g_{\perp}\right)$. Thus, the copperoxygen distances parallel and perpendicular to the $\mathrm{C}_{4}$ axis can be written in terms of the reference distance $R_{0}$ and the relative tetragonal elongation ratio $\tau$ as:

$$
R_{\|} R(1+2 \tau), \quad R_{\perp} R(1-\tau) .
$$

Now the measured d-d transition absorption bands $E-$ $1\left(=E_{x y}\right)$ in Ref. [13] at various concentrations $x$ are fitted in this section. Since 1) $E_{1}$ (or $10 D q$ ) shows the overall increase from $12376 \mathrm{~cm}^{-1}$ for $7.5 \mathrm{~mol} \%$ to $12484 \mathrm{~cm}^{-1}$ for $30 \mathrm{~mol} \%$ and medium fluctuations with $x$ and 2) the measured $g_{\|}$and $A_{\|}$also exhibit sine / cosine like variations of $x$ (see Fig. 3 of Ref. [13]). For convenience, the Fourier type function can be adopted to describe the above concentration variation of the cubic field parameter:

$$
D q=D q_{0}\{1+a \cos (\omega x)+b \sin (\omega x)\} .
$$

Here the adjustable coefficients $a, b$, and $\omega$ can be determined by fitting the experimental d-d transition bands. The reference value $D q_{0} \approx 1240 \mathrm{~cm}^{-1}$ may be obtained from the optical spectral measurements for $\mathrm{Cu}^{2+}$ in various oxide glasses [15]. By fitting the theoretical $E_{1}$ to the experimental data at various concentrations $x$ [13], the optimal quantities in Eq. (5) are obtained as:

$$
a \approx-0.0112, \quad b \approx 0.0082, \quad \omega \approx 1898 .
$$

The corresponding d-d transition bands $E_{1}$ are calculated as $12393,12546,12394$, and $12429 \mathrm{~cm}^{-1}$, which are comparable with the experimental values [13].

In the light of the perturbation formulas of EPR parameters in Eq. (1) and the expressions of the energy denominators in Eq. (2), similar but less significant fluctuations of orbital reduction factor $k$ than $D q$ can be expected because of the whole increasing $g$ factors (especially $g_{\|}$) and moderate fluctuations with $x$. For convenience, a factor $(-1 / 3)$ is added to the terms except 1 in the square bracket of Eq. (5). Then, the concentration dependence of the orbital reduction factor can be similarly expressed as the Fourier type function:

$$
k=k_{0}\left\{\frac{1-[a \cos (\omega x)-b \sin (\omega x)]}{3}\right\}
$$

Here the reference value $k_{0} \approx 0.812$ is obtained from the optical spectral analysis for $\mathrm{Cu}^{2+}$ in some oxide glasses [15]. Utilizing Eq. ( 7 ), $k \approx 0.805,0.811,0.820$ and 0.804 are calculated for $x=7.5,15,22.5$ and $30 \mathrm{~mol} \%$, respectively. 
The above values are qualitatively consistent with the averages ( $\sim 0.81-0.82[13])$ of the fitted bonding parameters $\alpha^{2}$ and $\beta 1^{2}$ in the previous studies and can be regarded as reasonable.

From Eq. (1), the anisotropy $\Delta g\left(=g_{\|}-g_{\perp}\right)$ is relevant to the denominator $E_{2}$ of $g_{\perp}$, which correlates closely with the tetragonal crystal-field parameters $D s$ and $D t$ and hence with the relative tetragonal elongation ratio $\tau$. From the overall decreasing (from 0.278 at $7.5 \mathrm{~mol} \%$ to 0.255 at $30 \mathrm{~mol}$ $\%)$ tendency and the slight fluctuations of the measured $\Delta g$ with $x$ [13], the relative elongation ratio can be expressed by the following Fourier type function:

$$
\tau \approx \tau_{0}\left\{\frac{1-[a \cos (\omega x)-b \sin (\omega x)]}{5}\right\}
$$

where $\tau_{0}$ is the reference value of the relative elongation ratio. Inputting the above values and the spin-orbit coupling coefficient $\zeta_{0}\left(\approx 829 \mathrm{~cm}^{-1}\right.$ [30]) for free $\mathrm{Cu}^{2+}$ into the $g$ formulas and matching the theoretical $\Delta g$ to the observed results, we have $\tau_{0} \approx 1.45 \%$. The corresponding tetragonal elongation ratios $\tau \approx 1.78 \%, 2.11 \%, 2.44 \%$ and $2.77 \%$ are obtained for $x=7.5,15,22.5$ and $30 \mathrm{~mol} \%$, respectively. The theoretical $g$ factors $\left(\mathrm{Cal}^{b}\right)$ for various concentrations $\mathrm{x}$ are shown in Table I. For comparison, the calculated $g$ factors $\left(\mathrm{Cal}^{a}{ }^{a}\right)$ based on the simple $g$ formulas and the fitted bonding parameters $\alpha^{2}, \beta 1^{2}$ and $\beta^{2}$ as well as the orbital reduction factor $K^{2}=0.77$ in the previous analysis [13] are also collected in Table I.

From Eq. (1), hyperfine structure constants depend mainly upon the isotropic contributions from the core polarization constant $\kappa_{0}$ and the anisotropic ones related to reduction factor $H$ or $g$-shifts. Because of absence of the perpendicular components of $A$ factors in Ref. [13], the concentration variations of $A_{\|}$may be attributed to the related concentration variations of the core polarization constant $\kappa$ and orbital reduction factor $k$ as well as the g-shifts $\left(g_{i}-g_{s}\right.$, with $i=\|$ and $\perp$ ). Thus, the concentration dependence of the core polarization constant can be similarly expressed as the Fourier type function:

$$
\kappa=\kappa_{0}\left\{\frac{1-[a \cos (\omega x)-b \sin (\omega x)]}{3}\right\} .
$$

Here $\kappa_{0}$ is the reference value, which is usually taken as the expectation value 0.3 for $3 \mathrm{~d}$ transition-metal ions in crystals [26]. The above expression yields $\kappa \approx 0.301,0.289$, 0.311 and 0.298 for $x=7.5,15,22.5$ and $30 \mathrm{~mol} \%$, respectively. The reduction factor can be fixed as 0.81 (i.e., about $20 \%$ reduction from the ideal value of unity in the absence of tetragonal elongation distortion) for the sake of reduction in the number of adjustable parameters. Substituting these values and the dipolar hyperfine coupling parameter $P_{0}\left(\approx 402 \times 10^{-4} \mathrm{~cm}^{-1}\right.$ [27]) for a free $\mathrm{Cu}^{2+}$ ion into Eq. (1), the concentration dependences of hyperfine structure constants $\left(\mathrm{Cal} .{ }^{b}\right)$ are obtained and compared with the experimental data in Table I.

\section{Results and discussion}

Table I displays that the calculated EPR parameters based on the above Fourier type functions of cubic field parameter, orbital reduction factor, relative tetragonal elongation ratio, and core polarization constant with concentration $x$ by using only three adjustable coefficients $(a, b$, and $\omega)$ for $\mathrm{Cu}^{2+}$ in TAW glasses show reasonable agreement with the observed results. Thus, the previous EPR results for the studied systems are suitably interpreted uniformly. Several points may be discussed here.

(1) The concentration variations of the d-d transition optical spectra reflect the modifications of the local crystal-field strength of the $\mathrm{Cu}^{2+}$ centers with the concentration variations of the modifier induced NBOs and the difference in field strength between $\mathrm{Ag}^{+} / \mathrm{W}^{6+}$ and $\mathrm{Te}^{4+}$ cations. First, the increases of the concentration of $\mathrm{WO}_{3}$ bring forward increasing NBOs with much higher polarizability, which mainly acts as the ligands of the $\mathrm{Cu}^{2+}$ dopants and leads to the fluctuations of the local crystal-fields. Second, when $\mathrm{Te}^{4+}$ is gradually replaced by $\mathrm{W}^{6+}$ with higher field strength, the original TeO-Te linkages in the glass network are partially substituted by the new Te-O-W or W-O-W ones through some $\mathrm{TeO}_{4}$ units transforming into $\mathrm{TeO}_{3}$ ones, as indicated by the 460 - $490 \mathrm{~cm}^{-1}$ bands in the IR measurements [13]. Third, the strength of the $\mathrm{Cu}-\mathrm{O}$ bonds in the $\left[\mathrm{CuO}_{6}\right]^{10-}$ groups may be

\begin{tabular}{|c|c|c|c|c|c|c|c|c|c|c|}
\hline \multirow[t]{2}{*}{$x(\mathrm{~mol} \%)$} & \multicolumn{3}{|c|}{$g_{\|}$} & \multicolumn{3}{|c|}{$g_{\perp}$} & \multicolumn{2}{|c|}{$A_{\|}$} & \multicolumn{2}{|c|}{$A_{\perp}$} \\
\hline & $\mathrm{Cal}^{a}{ }^{a}$ & Cal. $^{b}$ & Expt. [13] & $\mathrm{Cal}^{a}{ }^{a}$ & Cal. ${ }^{b}$ & Expt.[13] & Cal. ${ }^{b}$ & Expt. [13] & Cal. ${ }^{b}$ & Expt. \\
\hline 7.5 & 2.352 & 2.356 & $2.352(1)$ & 2.105 & 2.099 & $2.103(1)$ & -121.7 & $-124(0.5)$ & -0.7 & - \\
\hline 15 & 2.357 & 2.357 & $2.358(1)$ & 2.083 & 2.089 & $2.083(1)$ & -116.2 & $-113(0.5)$ & 2.9 & - \\
\hline 22.5 & 2.362 & 2.369 & $2.362(1)$ & 2.088 & 2.088 & $2.088(1)$ & -119.5 & $-118(0.5)$ & -3.2 & - \\
\hline 30 & 2.358 & 2.355 & $2.357(1)$ & 2.077 & 2.086 & $2.079(1)$ & -116.3 & $-114(0.5)$ & -0.1 & - \\
\hline
\end{tabular}
enhanced, because of the increasing density of TAG glasses

${ }^{a}$ Calculated $g$ factors based on the simple $g$ formulas using the adjustable bonding parameters $\alpha^{2}, \beta_{1}^{2}$, and $\beta^{2}$ as well as the orbital reduction factor $K^{2}=0.77$ in the previous work [13].

${ }^{b}$ Calculations based on the uniform perturbation formulas of the EPR parameters and the concentration dependences of the relevant quantities in this work. 
with $x$ [13]. This point can also be illustrated by the blue shifts of the IR bands in 650- 680 and $730-780 \mathrm{~cm}^{-1}$ regions, which reflects enhancing stretching vibrations of $\mathrm{TeO}_{3}$ units [13]. Thus, the overall increasing d-d transition bands with some fluctuations for $\mathrm{Cu}^{2+}$ in TAW glasses can be understood.

(2) The features of $g$ factors can be briefly analyzed here. The overall slightly increasing $g_{\|}$is attributed to the combined effects of the moderately increasing $k$ at two middle concentrations and the quiveringly increasing $D q$, and the former effect is slightly stronger. The highest $g_{\|}$at 22.5 mol\% is mainly attributable to the biggest $k$ and the moderate increase of $D q$ at this concentration. On the other hand, the slightly decreasing $g_{\perp}$ can be ascribed to the increasing relative elongation ratio $\tau$ and hence the increasing magnitude of the negative tetragonal crystal-field parameter $D s$, leading to the increasing denominator $E_{2}$. Further, the whole increasing anisotropy $\Delta g$ (related to the nearly unchanging $g_{\|}$and the slightly decreasing $g_{\perp}$ ) is consistent with the increasing relative elongation ratio $\tau$. Finally, although the theoretical $g_{\|}$ and $g_{\perp}\left(\mathrm{Cal}^{a}\right)$ based on the simple $g$ formulas and the fitted bonding parameters $\alpha^{2}, \beta 1^{2}$ and $\beta^{2}$ (which were even largely higher than unity) and the orbital reduction factor $K^{2}=0.77$ in Ref. [13] show good agreement with the experimental data. Present calculations can be regarded as an improvement to the previous studies given the inclusion of the detailed local structures around the $\mathrm{Cu}^{2+}$ dopants, adoption of fewer adjustable parameters, and uniform treatments of both $g$ factors and hyperfine structure constants. Interestingly, the present orbital reduction factors $k(\approx 0.805,0.811,0.820$ and 0.804$)$ from Eq. (7) show largely consistent tendency with the averages $\left(\approx 0.809,0.823,0.824\right.$ and 0.821 [13]) of the fitted $\alpha^{2}$ and $\beta 1^{2}$ at $7.5,15,22.5$ and $30 \mathrm{~mol} \%$, respectively, and the same maximum at $22.5 \mathrm{~mol} \%$. So, present calculations can be regarded as suitable in physics, which conformably reveal the overall weakening covalency with the weakest covalency of the copper-oxygen bonds at $22.5 \mathrm{~mol} \%$. Physically, the concentration dependence of covalency can be ascribed to the modification of the electronic cloud distribution around the copper dopants. For example, the electronic cloud density around oxygen ions at the middle concentrations (especially $22.5 \%$ ) can be enhanced and lead to the significant decline of the $\mathrm{Cu}^{2+}-\mathrm{O}^{2-}$ orbital admixtures, yielding the weakest covalency and highest $k$.

(3) The studied $\mathrm{Cu}^{2+}$ centers in TAG glasses exhibit the tetragonal elongations of about $2-3 \%$ due to the Jahn-Teller effect. Interestingly, analogous moderate tetragonal elongation ratios $\left(\approx 3 \%\right.$ and $4 \%$ ) were also found for $\mathrm{Cu}^{2+}$ in $\mathrm{xLi}_{2} \mathrm{O} \cdot\left(30_{-x}\right) \cdot \mathrm{K}_{2} \mathrm{O} \cdot 70 \mathrm{~B}_{2} \mathrm{O}_{3}(0 \leq x \leq 25 \mathrm{~mol} \%)$ [28] or $40 \mathrm{MgO} \cdot\left(10_{-x}\right) \mathrm{PbF}_{2} \cdot 50 \mathrm{SiO}_{2-x} \mathrm{CuO}(0.1 \leq x \leq 1.0 \mathrm{~mol} \mathrm{\%})$ [29] and $\left(70_{-x}\right) \mathrm{Bi}-2 \mathrm{O}_{3-x} \mathrm{Li}_{2} \mathrm{O}-30\left(\mathrm{ZnO}-\mathrm{B}_{2} \mathrm{O}_{3}\right)(0 \leq x \leq$ $20 \mathrm{~mol} \%$ ) glasses [30]. The nearly linearly increasing relative elongation ratios $\tau$ (from 1.78 to $2.77 \mathrm{~mol} \%$ ) can re- flect the moderate tetragonal elongations around the copper dopants in TAW glasses. The increasing tendency of the relative elongation can be ascribed to the enhancing vibrational interactions due to the strengthened copper-oxygen bonding (i.e., the increasing $D q$ ) with increasing $x$. Thus, the relative tetragonal elongation ratio and its concentration dependence obtained in this work by analyzing the $g$ anisotropy for $\mathrm{Cu}^{2+}$ in TAW glasses can be regarded as reasonable.

(4) The magnitude of hyperfine structure constant $A_{\|}$ exhibits an overall decreasing rule with slight fluctuations, yielding a slight decrease at $15 \mathrm{~mol} \%$. This can be illustrated as the competitive influences from the overall decreasing $\kappa$, $k$, and $g_{\perp}-g_{s}$ under the fixed reduction factor $H$. The obvious decline of the magnitude for $A_{\|}$at $15 \mathrm{~mol} \%$ with respect to the other concentrations is mainly attributed to the significant decrease (by 4\%) of the core polarization constant. This point is ascribed to the decrease of the electronic cloud density around copper arising from the enhancing electronic cloud of oxygen, leading to the weakest isotropic copper $3 \mathrm{~d}$ $3 \mathrm{~s}(4 \mathrm{~s})$ admixtures and hence the lowest $\kappa$ at this concentration. Normally, the hyperfine structure constants in the EPR measurements are given as the absolute values [13]. From present calculations, both $A_{\|}$and $A_{\perp}$ are negative, because of the dominant-negative contributions relevant to $\kappa$ and the much smaller positive contributions relevant to $g$-shifts. Of course, the present calculated $A_{\perp}$ with the much smaller magnitudes remain to be further verified with additional EPR experiments.

\section{Summary}

The local structures and EPR parameters for $\mathrm{Cu}^{2+}$ in TAW glasses at distinct modifier concentrations $\mathrm{x}$ are theoretically investigated using the perturbation formulas of these parameters. Subject to the Jahn-Teller effect, the impurity $\mathrm{Cu}^{2+}$ centers are found to experience moderate tetragonal elongations $(\tau \approx 1.78-2.77 \%$ ). Based on merely three adjusted coefficients $a, b$, and $\omega$, the relevant model parameters $(D q, k, \tau$, and $\kappa$ ) are expressed in terms of the Fourier type functions of the modifier concentration. The observed concentration variations of the d-d transition bands and the EPR parameters are suitably interpreted in a uniform way. And the mechanisms of the above concentration dependences of these quantities are illustrated by the modifications of the local structure and the electron cloud distribution around the $\mathrm{Cu}^{2+}$ dopant with the concentration $x$.

\section{Acknowledgments}

This work is financially supported by the Innovation Seedling Project of Sichuan Province (No.2018100) and a major project of CDNU (No.CS18ZDZ0511). 
1. A. Palui and A. Ghosh, Structure and dielectric properties of $\mathrm{Ag}_{2} \mathrm{O}-\mathrm{SeO}_{2}-\mathrm{TeO}_{2}$ mixed former glasses, J. Non. Cryst. Solids. 482 (2018) 230. https://doi.org/10.1016/ JNonCrystSolids.482.230

2. B. V. R. Chowdari and P. Pramoda Kumari, Thermal, electrical and XPS studies of $\mathrm{Ag}_{2} \mathrm{OTeO}_{2} \mathrm{P}_{2} \mathrm{O}_{5}$ glasses, J. Non. Cryst. Solids. 197 (1996) 31. https://doi.org/10. 1016/JNonCrystSolids.197.31

3. Yasuhiko Iwadate, Mineta Suzuki, Takeo Hattori, Kazuko Fukushima and Keiji Itoh, Evolution of local structure in $\mathrm{Ag}_{2} \mathrm{O}-\mathrm{TeO}_{2}$ glasses with addition of $\mathrm{Ag}_{2} \mathrm{O}$ analyzed by pulsed neutron diffraction and Raman spectroscopy, J. Alloys Compd. 389 (2005) 229. https://doi.org/10.1016/ JAlloys Compd.389.229

4. D. Linda, M. Dutreilh-Colas, D. Hamani, P. Thomas and A. Kabadou, Temperature dependent structural and spectroscopic studies of sodium gallosilicate nitrite sodalite, Mat. Res. Bull. 45 (2010) 1816. (|https://doi.org/10.1016/jMatRes Bull.45.1816)

5. D. Linda, J.-R. Duclére, T. Hayakawa, M. Dutreilh-Colas and P. Thomas, Optical properties of tellurite glasses elaborated within the $\mathrm{TeO}_{2}-\mathrm{Tl}_{2} \mathrm{O}-\mathrm{Ag}_{2} \mathrm{O}$ and $\mathrm{TeO}_{2}-\mathrm{ZnO}-\mathrm{Ag}_{2} \mathrm{O}$ ternary systems, J. Alloys Compd. 561 (2013) 151. https://doi. org/10.1016/JAlloysCompd.561.151

6. K. Kato, T. Hayakawa, and Y. Kasuya, Influence of $\mathrm{Al}_{2} \mathrm{O}_{3}$ incorporation on the third-order nonlinear optical properties of $\mathrm{Ag}_{2} \mathrm{O}-\mathrm{TeO}_{2}$ glasses, J. Non. Cryst. Solids. 431 (2016) 97. https://doi.org/10.1016/ JNonCrystSolids.431.97

7. S. E. Ibrahim, Y. S. Rammah, I. Z. Hager ans R. E. Mallawany, UV and electrical properties of $\mathrm{TeO}_{2}-\mathrm{WO}_{3}$ $\mathrm{Li}_{2} \mathrm{O}-\mathrm{Nb}_{2} \mathrm{O}_{5} / \mathrm{Sm}_{2} \mathrm{O}_{3} / \mathrm{Pr}_{6} \mathrm{O}_{11} / \mathrm{Er}_{2} \mathrm{O}_{3}$ glasses, J. Non. Cryst. Solids. 498 (2018) 443. https://doi.org/10.1016/ JNonCrystSolids.498.443

8. M. Reda Zaki, D. Hamani, M. Dutreilh-Colas, J.-R. Duclére, Synthesis, thermal, structural and linear optical properties of new glasses within the $\mathrm{TeO}_{2}-\mathrm{TiO}_{2}-\mathrm{WO}_{3}$ system, J. Non. Cryst. Solids. 484 (2018) 139. https://doi.org/10.1016/ JNonCrystSolids.484.139

9. T. Subrahmanyam, K. Rama Gopal, R. P. Suvarna, B. C. Jamalaiah and M. V. V. Kumar, Luminescent properties of $\mathrm{Tb}^{3+}$. doped $\mathrm{TeO}_{2-} \mathrm{WO}_{3-} \mathrm{GeO}_{2}$ glasses for green laser applications, Opt. Mater. 80 (2018) 154. https: //doi.org/10.1016/ OptMater.80.154

10. D. K. Gaikwad, S. S. Obaid, M. I. Sayyed, R. R. Bhosale and P. P. Pawar, Comparative study of gamma ray shielding competence of $\mathrm{WO}_{3-} \mathrm{TeO}_{2-} \mathrm{PbO}$ glass system to different glasses and concretes, Mat. Chem. Phys. 213 (2018) 508. https: //doi.org/10.1016/MatChemPhys.213.508

11. M. I. Sayyed, Saleem I. Qashou and Z. Y. Khattari, Radiation shielding competence of newly developed $\mathrm{TeO}_{2}-\mathrm{WO}_{3}$ glasses, J. Alloys Compd. 696 (2017) 632. https: / / doi.org/10. $1016 / \mathrm{JAlloysCompd.696.632}$

12. A. E. Ersundu, M. Büyükyıldız, M. Çelikbilek Ersundu, E. Şakar and M. Kurudirek, The heavy metal oxide glasses within the $\mathrm{WO}_{3-} \mathrm{MoO}_{3}-\mathrm{TeO}_{2}$ system to investigate the shielding properties of radiation applications, Prog. Nucl.
Energy, 104 (2018) 280. https://doi.org/10.1016/ ProgNuclEnergy104.280

13. G. Upender, C. Sameera D, V. Kamalaker and V. C. Mouli, The structural and spectroscopic investigations of ternary tellurite glasses, doped with copper, Journal of Alloys and Compounds, $\mathbf{5 0 9}$ (2011) 5887. https://doi.org/10.1016/ JAlloysCompd.509.5887

14. S. Sugano, Y. Tanabe, and H. Kamimura, Multiplets of Transition-Metal Ions in Crystals, Academic Press, (USA, 1970).

15. A. S. Chakravarty, Introduction to the magnetic properties of solids, Wiley-Interscience Publication, (USA, 1980).

16. M. Q. Kuang, S. Y. Wu, B. T. Song and Z. H. Zhang, Theoretical studies on the local structure and spin Hamiltonian parameters for the orthorhombic $\mathrm{Cu}^{2+}$ center in $\mathrm{LiNbO}_{3}$, Optik, $\mathbf{1 2 3}$ (2012) 1601. https://doi.org/10.1016/opt.123. 1601

17. C. C. Ding, S. Y. Wu, G. L. Li and Y. Q. Xu, Investigations on the defect structure and the spin Hamiltonian parameters for $\mathrm{LaSrGa}_{0.995} \mathrm{Cu}_{0.005} \mathrm{O}_{4}$ crystal, Phys. B, 459 (2015) 129. https://doi.org/10.1016/PhysB.459.129

18. M. Q. Kuang, S. Y. Wu, X. F. Hu, and B. T. Song, Theoretical Investigations of the Knight Shifts and the Hyperfine Structure Constants for the Tetragonal $\mathrm{Cu}^{2+}$ Sites in the Bismuth- and Thallium-Based High-Tc Superconductors, $Z$. Naturforsch. A. 68 (2013) 442. https://DOI:10.5560/ ZNA.2013-0019/ZNA.68.442

19. S. Y. Wu, H. M. Zhang, P. Xu, and S. X. Zhang, Studies on the defect structure for $\mathrm{Cu}^{2+}$ in CdSe nanocrystals, Spect. Acta A, 75 (2010) 230. https://doi.org/10.1016/ SpecActaA. 75.230

20. H. M. Zhang, S. Y. Wu, M. Q. Kuang, and Z. H. Zhang, Investigation of the EPR parameters and local structures for $\mathrm{Cu}^{2+}$ in Bis(l-asparaginato) $\mathrm{M}(\mathrm{II})$ catalysts $(\mathrm{M}=\mathrm{Zn}, \mathrm{Cd}, \mathrm{Mg})$, J. Phys. Chem. Solids, 73 (2012) 846. https : //doi .org/ 10.1016/JPhyChemSol.73.846

21. M. T. Barriuso, J. A. Aramburu, and M. Moreno, Puzzling absence of hyperfine interaction in the $\mathrm{D}_{4 h} \mathrm{RhCl}_{6}^{4-}$ centre: role of the $4 \mathrm{~d}-5 \mathrm{~s}$ hybridization in $\mathrm{Rh}^{2+}$ centres, J. Phys.: Cond. Matt. 14 (2002) 6521.https: / / iopscience.iop.org/ article/10.1088/JPhysCondMatt.14.6521

22. D. J. Newman and B. $\mathrm{Ng}$, The superposition model of crystal fields, Rep. Prog. Phys. 52 (1989) 699. https: //iopscience.iop.org/article/10.1088/ 0034-4885/52/6/002/RepProgPhys.52.699

23. Y.X. Hu, S.Y. Wu and X.F. Wang, Spin Hamiltonian parameters and local structures for tetragonal and orthorhombic $\mathrm{Ir}^{2+}$ centers in $\mathrm{AgCl}$, Phil. Mag. 90 (2010) 1391. https : //doi. org/10.1080/PhilMag.90.1391

24. H. M. Zhang, S. Y. Wu, P. Xu and L. L. Li, Investigations on the Spin Hamiltonian Parameters and the Local Structures for Various $\mathrm{Rh}^{2+}$ Centers in $\mathrm{NaCl}$, Z. Naturforsch. A. 65 (2010) 591. https: //www. znaturforsch.com/ s65a//ZNatA.65.591

25. Z. H. Zhang, S. Y. Wu, M. Q. Kuang and B. T. Song, Theoretical studies of the local structures and the $g$ factors for the tetragonal $\mathrm{Ti}^{3+}-$ Vo centers in $\mathrm{BaTiO}_{3}$ bulks and thin films, Eur. 
Phys. J. Appl. Phys. 56 (2011)10303. https://doi.org/ $10.1051 /$ EurPhys JApplPhys.56.10303

26. J. S. Griffith, The Theory of Transition-Metal Ions, Cambridge University Press, (UK, 1964).

27. B. R. McGarvey, Anisotropic Hyperfine Interaction of $\mathrm{Cr}^{53}$ in Chromium (III) Acetylacetonate, J. Chem. Phys. 71 (1967) 51. https://doi.org/10.1063/JChemPhys.71.51

28. H. M. Zhang, S. Y. Wu and Z. H. Zhang, Theoretical studies of the spin Hamiltonian parameters and the local structures for the tetragonal $\mathrm{Cu}^{2+}$ centers in the LKB glasses, J. Non. Cryst. Solids. 357 (2011) 2054. https://doi.org/10.1016/ JNonCrystSolids.357.2054
29. C. C. Ding, S. Y. Wu, X. F. Hu, G. L. Li and Y. Q. Xu, An investigation of the local distortions and the EPR parameters for $\mathrm{Cu}^{2+}$ in $40 \mathrm{MgO}-(10-\mathrm{x}) \mathrm{PbF}_{2}-50 \mathrm{SiO}_{2-x} \mathrm{CuO}$ glasses, J. Alloys Compd. 664 (2016) 250. https://doi.org/10.1016/ JAlloysComd.664.250 J

30. F. Zhang, S. Y. Wu, Q. Q. Tan, Q. Zhang and G. J. Chen, Calculations of defect structures and spin Hamiltonian parameters of copper in $\left(70_{-x}\right) \mathrm{Bi}_{2} \mathrm{O}_{3-x} \mathrm{Li}_{2} \mathrm{O}_{-30}(\mathrm{ZnO}-$ $\mathrm{B}_{2} \mathrm{O}_{3}$ ) glasses at different compositions, Mod. Phys. Lett. B, 32 (2018) 1850305. https://doi.org/10.1142/ ModPhysLettB.32.1850305 